\title{
Probing light charged Higgs at the LHC Run 2
}

\author{
Abdesslam Arhrib \\ Faculté des Sciences et Techniques, Abdelmalek Essaadi University, B.P. 416, Tangier, Morocco. \\ E-mail: aarhrib@gmail.com
}

\section{Rachid Benbrik*}

MSISM Team, Faculté Polydisciplinaire de Safi, Sidi Bouzid, B.P. 4162, Safi, Morocco.

LPHEA, FSSM, Cadi Ayyad University, Marrakech, Morocco

E-mail: r.benbrikauca.ac.ma

\section{Abdessamad Rouchad}

MSISM Team, Faculté Polydisciplinaire de Safi, Sidi Bouzid, B.P. 4162, Safi, Morocco.

LPHEA, FSSM, Cadi Ayyad University, Marrakech, Morocco

E-mail: abdessamad.rouchad@edu.uca.ac.ma

In this contribution we discuss light charged Higgs signatures in two-Higgs-doublet-model (2HDM) type-1 and type-X. We determine the allowed regions by including several theoretical constraints including elctroweak precision and flavour observables as well as experimental constraints coming from Higgs data Run-2 and from non-observed additional Higgs at Run-2 of LHC. Our proposal scenarios shows that the production and decay of charged Higgs in 2HDM type- 1 and type-X are complementary depending on the assumptions of the 2HDM parameters. Additionally, we find that in a wide range of parameter space in which non-standard neutral Higgs boson $h$ of about $95 \mathrm{GeV}$ can change dramatically the decay of light charged Higgs.

Seventh International Workshop

Prospects for Charged Higgs

Discovery at Colliders

25-28 Septembre 2018

Uppsala, Sweden

\footnotetext{
* Speaker.
} 


\section{Itroduction}

Recent discovery of a scalar particle at the Large Hadron Collider (LHC)[1, 2] together with various successful experiments has established the Standard Model (SM) as the fondamental theory of strong and electroweak interactions. Despite its great success in explaining all available data, the SM has serious deficiencies and there are several phenomenological indications that all questions cannot find an answer in the SM. The arguments supporting an extension of the SM is then well motivated.

The Two-Higgs-Doublet Model (2HDM) is one of the simplest beyond the SM extension of the Higgs mechanism of the electroweak symmetry breaking which may arise naturally in the scalar sector of various theories. In this model, we introduce two doublets of scalar fields $\Phi_{1}$ and $\Phi_{2}$. This simple extension contains very interesting charged Higgs sector. Adding $\Phi_{2}$ in the $2 \mathrm{HDM}$ potetial increases the numbers of free parameters substantially which lead to additional degrees of freedom in the scalar sector. Furthermore, we left with three massive neutral scalars $h, H$ and $A$ in the CP-conserving limit and a charged Higgs boson $H^{ \pm}$. One of the neutral Higgs bosons can be identified as SM-like with mass at $125 \mathrm{GeV}$. In order to avoid tree-level flavour-changing neutral currents from the Yukawa interaction several symmertries are imposed in the 2HDM. As a result different versions of 2HDM exist and labelled as type-1, type-2, type-X and type-Y.

Charged Higgs bosons pose a special challenge for experimental searches. They are dominantly produced in association with top quarks $\left(t b H^{ \pm}\right)$. The $H^{ \pm} \rightarrow t b$ holds a little chance for discovering charged Higgses at the LHC due the large luminosity. In Ref we pointed a potential decay modes $H^{ \pm} t o W A$ and $W h$. In this contribution we propose benchmark points for charged Higgs decays at the LHC Run-2, taking into account several existing contraints on the parameter space of 2HDM type- 1 and type-X. The contribution is organized as follows. In section.2, we introduce a brief review oh $2 \mathrm{HDM}$ and explore the benchmarks points.

\section{The 2HDM}

The most general scalar potential which is $S U(2)_{L} \otimes U(1)_{Y}$ invariant is given by [3, 4]

$$
\begin{aligned}
V\left(\Phi_{1}, \Phi_{2}\right) & =m_{1}^{2} \Phi_{1}^{\dagger} \Phi_{1}+m_{2}^{2} \Phi_{2}^{\dagger} \Phi_{2}-\left(m_{12}^{2} \Phi_{1}^{\dagger} \Phi_{2}+\text { h.c }\right)+\frac{1}{2} \lambda_{1}\left(\Phi_{1}^{\dagger} \Phi_{1}\right)^{2} \\
& +\frac{1}{2} \lambda_{2}\left(\Phi_{2}^{\dagger} \Phi_{2}\right)^{2}+\lambda_{3}\left(\Phi_{1}^{\dagger} \Phi_{1}\right)\left(\Phi_{2}^{\dagger} \Phi_{2}\right)+\lambda_{4}\left(\Phi_{1}^{\dagger} \Phi_{2}\right)\left(\Phi_{1}^{\dagger} \Phi_{2}\right) \\
& +\left[\frac{\lambda_{5}}{2}\left(\Phi_{1}^{\dagger} \Phi_{2}\right)^{2}+\text { h.c. }\right] .
\end{aligned}
$$

The complex (pseudo)scalar doublets $\Phi_{i}(i=1,2)$ can be parameterized as

$$
\Phi_{i}(x)=\left(\begin{array}{c}
\phi_{i}^{+}(x) \\
\frac{1}{\sqrt{2}}\left[v_{1}+\rho_{1}(x)+i \eta_{1}(x)\right]
\end{array}\right),
$$

with $v_{1,2} \geq 0$ being the VEVs satisfying $v=\sqrt{v_{1}^{2}+v_{2}^{2}}$, with $v=246.22 \mathrm{GeV}$. Hermiticity of the potential forces $\lambda_{1,2,3,4}$ to be real while $\lambda_{5}$ and $m_{12}^{2}$ can be complex. In this work we choose to work in a CP-conserving potential where both VEVs are real and so are also $\lambda_{5}$ and $m_{12}^{2}$. 


\begin{tabular}{||l|l|l|l|l|l|l|l|l|l||}
\hline \hline & $\kappa_{u}^{h}$ & $\kappa_{d}^{h}$ & $\kappa_{l}^{h}$ & $\kappa_{u}^{H}$ & $\kappa_{d}^{H}$ & $\kappa_{l}^{H}$ & $\kappa_{u}^{A}$ & $\kappa_{d}^{A}$ & $\kappa_{l}^{A}$ \\
\hline Type-1 & $c_{\alpha} / s_{\beta}$ & $c_{\alpha} / s_{\beta}$ & $c_{\alpha} / s_{\beta}$ & $s_{\alpha} / s_{\beta}$ & $s_{\alpha} / s_{\beta}$ & $s_{\alpha} / s_{\beta}$ & $c_{\beta} / s_{\beta}$ & $-c_{\beta} / s_{\beta}$ & $-c_{\beta} / s_{\beta}$ \\
\hline Type-X & $c_{\alpha} / s_{\beta}$ & $c_{\alpha} / s_{\beta}$ & $-s_{\alpha} / c_{\beta}$ & $s_{\alpha} / s_{\beta}$ & $s_{\alpha} / s_{\beta}$ & $c_{\alpha} / c_{\beta}$ & $c_{\beta} / s_{\beta}$ & $-c_{\beta} / s_{\beta}$ & $s_{\beta} / c_{\beta}$ \\
\hline
\end{tabular}

Table 1: Yukawa couplings in terms of mixing angles in the two proposed 2HDM Types.

After EWSB, three of the eight degrees of freedom in the Higgs sector of the 2HDM are eaten by the Goldstone bosons $\left(G^{ \pm}\right.$and $G$ ) to give masses to the longitudinal gauge bosons ( $W^{ \pm}$and $Z$ ). The remaining five degrees of freedom becomes the aforementioned physical Higgs bosons. After using the minimization conditions for the potential together with the $W^{ \pm}$boson mass requirement, we end up with seven independent parameters which will be taken as

$$
m_{h}, m_{H}, m_{A}, m_{H^{ \pm}}, \alpha, \tan \beta, m_{12}^{2},
$$

where, as usual, $\tan \beta \equiv v_{2} / v_{1}$ and $\beta$ is also the angle that diagonalizes the mass matrices of both the $\mathrm{CP}$-odd and charged Higgs sector while the angle $\alpha$ does so in the $\mathrm{CP}$-even Higgs sector.

The most commonly used version of a CP-conserving 2HDM is the one which satisfy a discrete $Z_{2}$ symmetry $\Phi_{i} \rightarrow(-1)^{i+1} \Phi_{i}(i=1,2)$, that, when extended to the Yukawa sector, guarantees the absence of Flavor Changing Neutral Currents (FCNCs). Such a symmetry would also require $m_{12}^{2}=0$, unless we tolerate a soft violation of this by the dimension two term $m_{12}^{2}$ (as we do here). The Yukawa Lagrangian can then be written as

$$
-\mathscr{L}_{Y}=\bar{Q}_{L}\left(Y_{1}^{d} \Phi_{1}+Y_{2}^{d} \Phi_{2}\right) d_{R}+\bar{Q}_{L}\left(Y_{1}^{u} \tilde{\Phi}_{1}+Y_{2}^{u} \tilde{\Phi}_{2}\right) u_{R}+\bar{L}_{L}\left(Y_{1}^{l} \Phi_{1}+Y_{2}^{l} \Phi_{2}\right) l_{R}+\text { h.c. },
$$

where $Q_{L}^{T}=\left(u_{L}, d_{L}\right)$ and $L_{L}^{T}=\left(l_{L}, l_{L}\right)$ are the left-handed quark doublet and lepton doublet, respectively, the $Y_{k}^{f}$ 's $(k=1,2$ and $f=u, d, l)$ denote the $3 \times 3$ Yukawa matrices and $\tilde{\Phi}_{k}=i \sigma_{2} \Phi_{k}^{*}$ $(k=1,2)$. Since the mass matrices of the quarks and leptons are a linear combination of $Y_{1}^{f}$ and $Y_{2}^{f}$, $Y_{1,2}^{d, l}$ and $Y_{1,2}^{u}$ cannot be diagonalized simultaneously in general. Therefore, neutral Higgs Yukawa couplings with flavor violation appear at tree-level and contribute significantly to FCNC processes such as $\Delta M_{K, B, D}$ as well as $B_{d, s} \rightarrow \mu^{+} \mu^{-}$mediated by neutral Higgs exchanges. To avoid having those large FCNC processes, one known solution is to extend the $Z_{2}$ symmetry to the Yukawa sector. When doing so, we end up with the already discussed four possibilities regarding the Higgs bosons couplings to fermions [4].

After EWSB, the Yukawa Lagrangian can be expressed in the mass eigenstate basis as follows $[5,6]$ :

$$
\begin{aligned}
\mathscr{L}_{Y}= & -\sum_{f=u, d, \ell} \frac{m_{f}}{v}\left(\xi_{h}^{f} \bar{f} f h+\xi_{H}^{f} \bar{f} f H-i \xi_{A}^{f} \bar{f} \gamma_{5} f A\right) \\
& -\left(\frac{\sqrt{2} V_{u d}}{v} \bar{u}\left(m_{u} \xi_{A}^{u} P_{L}+m_{d} \xi_{A}^{d} P_{R}\right) d H^{+}+\text {h.c. }\right) .
\end{aligned}
$$

We give in Tab. 1, the values of the Yukawa couplings in the 2HDM type-1 and type-X. The couplings of $h$ and $H$ to gauge bosons $V=W^{ \pm}, Z$ are proportional to $\sin (\beta-\alpha)$ and $\cos (\beta-\alpha)$, respectively. Since these are gauge couplings, they are the same for all Yukawa types. The 2HDM possesses two alignment limits: one with $h$ SM-like[7, 8] and an other one with $H$ SM-like[9, 10]. 
In the present study, we will take into account two scenarios. For the first one we assume that $h$ is the SM-like Higgs boson discovered at CERN, which implies that $\cos (\beta-\alpha) \approx 0$ and in the second one we will focus on the alignment limit where $H$ is the SM-like in this case the limit $\sin (\beta-\alpha) \approx 0$ seems to be favored by LHC data.

\section{Light charged Higgs Boson}

Light charged Higgs states, i.e, with $m_{H \pm} \leq m_{t}-m_{b}$, are copiously induced by $t \bar{t}$ production followed by the top decay $t \rightarrow b H^{+}$(or the equivalent antitop mode). When kinematically allowed, $p p \rightarrow t \bar{t} \rightarrow b \bar{b} H^{-} W^{+}+$c.c. provides the most important source of light charged Higgs bosons, above and beyond the yield of various direct production modes: $g b \rightarrow t H^{-}$and $g g \rightarrow t \bar{b} H^{-}$[14], $g g \rightarrow W^{ \pm} H^{\mp}$ and $b \bar{b} \rightarrow W^{ \pm} H^{\mp}[15], q \bar{q}^{\prime} \rightarrow \phi H^{ \pm}$where $\phi$ denotes one of the three neutral Higgs bosons [16], $g g \rightarrow H^{+} H^{-}$and $q \bar{q} \rightarrow H^{+} H^{-}$[17], $q b \rightarrow q^{\prime} H^{+} b$ [18] and $c \bar{s}, c \bar{b} \rightarrow H^{+}$[19]. (See also Refs. $[20,21]$ for a review of all available $H^{ \pm}$hadro-production modes in 2HDMs.)

Searches for light charged Higgs boson with mass $m_{H^{ \pm}}<m_{t}$ have been performed by both ATLAS[22, 23, 27, 26, 25, 24] and CMS[28, 29, 30, 31]. The most relevant constraints in our scenarios are the constraints on the production of a light charged Higgs via top quark decay, $t \rightarrow$ $H^{+} b$, with subsequent decays $H^{ \pm} \rightarrow \tau^{ \pm} v[22,23,27,25,30,31], H^{+} \rightarrow c \bar{s}[26,29]$ and $H^{+} \rightarrow$ $c \bar{b}[28]$.

\section{Parameter space scans and constraints}

We numerically scanned the parameters of 2HDM-I and 2HDM-X using the public program 2HDMC [13] in the ranges:

$$
m_{h} / 2 \leq m_{A} \leq 300 \mathrm{GeV}, \quad, 90 \mathrm{GeV} \leq m_{H^{ \pm}} \leq 200 \mathrm{GeV},-1 \leq \cos (\beta-\alpha) \leq 1 \quad 0.5 \leq \tan \beta \leq 30 .
$$

with $m_{h}=95 \mathrm{GeV}$ and $m_{H}=125 \mathrm{GeV}$,

During the scan, each sampled model point was subjected to the following conditions:

- Unitarity, perturbativity, and vacuum stability enforced through the default 2HDMC method.

- The oblique parameters $\mathrm{S}, \mathrm{T}$ and $\mathrm{U}$ were calculated with $2 \mathrm{HDMC}$ methods and were required to fall within 95\% Confidence Level (CL) ellipsoid on 2018 PDG values[32], $S=0.02 \pm 0.10$, $T=0.07 \pm 0.12$ and $U=0.00 \pm 0.09$ with correlations $\rho_{S T}=0.92, \rho_{S U}=-0.66$ and $\rho_{T U}=$ -0.86 .

- All scalars in the models satisfied all (95\% CL) constraints included in the program HiggsBounds 5.2.0[33], as well as the requirement that one of the neutral scalar states should match the properties of the observed SM-like Higgs boson and we guarantee that with HiggsSignals[34] code.

- Satisfaction of the 95\% CL limits on b-physics observables calculated with the public code SuperIso-v3.6[35]. 

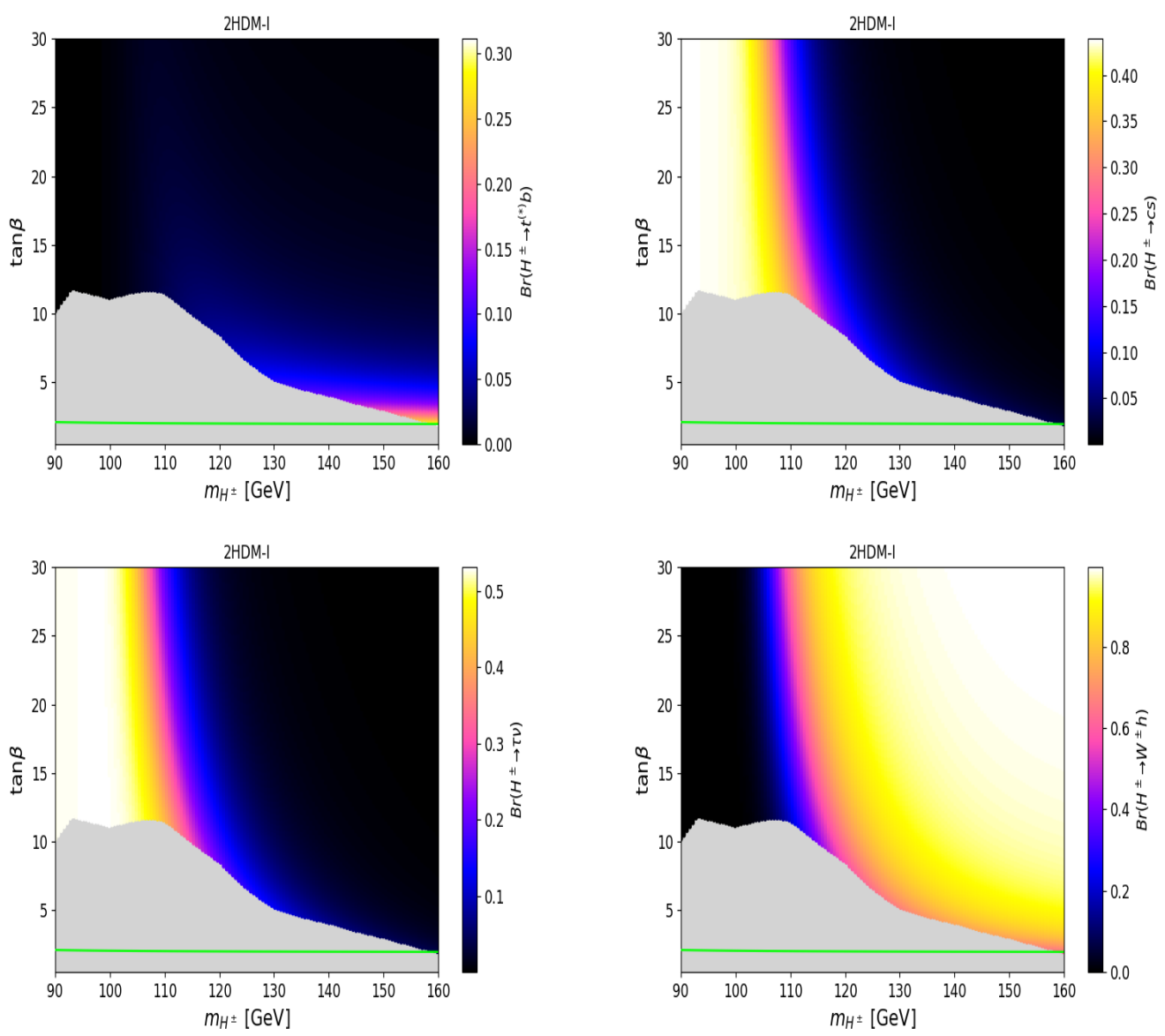

Figure 1: The $\operatorname{Br}\left(H^{ \pm} \rightarrow X Y\right)$ in the 2HDM-I mapped over the $\left(m_{H^{ \pm}}, \tan \beta\right)$ plane with $\sin (\beta-\alpha)=-0.13$, $m_{A}=169 \mathrm{GeV}, m_{h}=95 \mathrm{GeV}$ and $m_{H}=125 \mathrm{GeV}$. For $X Y \equiv t^{*} \bar{b}$ (top left), $X Y \equiv c \bar{s}$ (top right), $X Y \equiv \tau \nu$ (bottom left) and $X Y \equiv W^{ \pm *} h$.

\section{Results and discussions}

In this contribution, we activate the following set $m_{h}=95 \mathrm{GeV}$ and $m_{H}=125 \mathrm{GeV}$. This scenario is well motivated from the experimetal of view. In fact, looking few events around $95 \mathrm{GeV}$ is very exiting and it must be investigated in $2 \mathrm{HDM}$. The best-point is given in table. 4 with light $\tan \beta$ in 2HDM-type-1 and intermidiate values in 2HDM-type-X. It is clear from table.4 that $B R\left(H^{\rightarrow} W^{+} h\right)$ is maximal. Similary, we investigate this scenario and show our finding in Figure.(1), where the $B r\left(H^{ \pm} \rightarrow X Y\right)$ in the 2HDM-I are mapped over the $\left(m_{H^{ \pm}}, \tan \beta\right)$ plane with $\sin (\beta-\alpha)=-0.13, m_{A}=169 \mathrm{GeV}, m_{h}=95 \mathrm{GeV}$ and $m_{H}=125 \mathrm{GeV}$. For $X Y \equiv t^{*} \bar{b}$ (top left), $X Y \equiv c \bar{s}$ (top right), $X Y \equiv \tau \nu$ (bottom left) and $X Y \equiv W^{ \pm *} h$ and alternavely the predicted cross section $\sigma(p p \rightarrow t \bar{t}) \times B r\left(t \rightarrow H^{+} b\right) \times B r\left(H^{ \pm} \rightarrow W^{ \pm} h\right)$ in the 2HDM-1. Not like MSSM, where the situation of $95 \mathrm{GeV}$ is not possible, $2 \mathrm{HDM}$ type- 1 and type- $\mathrm{X}$ present an very important alternative for explainning $95 \mathrm{GeV}$ and being consistent with recent data. Again 2HDM type-1 is the most promising framework in which data can explain various observables. 


\begin{tabular}{|c|c|c|}
\hline \hline Parameters & 2HDM-I & 2HDM-X \\
\hline \hline$(\sin (\beta-\alpha), \tan \beta)$ & $(-0.130451 ; 4.6)$ & $(-0.00163 ; 11.98158)$ \\
$\left(m_{A}, m_{h}\right)[\mathrm{GeV}]$ & $(168.94 ; 95)$ & $(161.37 ; 95)$ \\
$\left(m_{H^{ \pm}}\right)[\mathrm{GeV}]$ & $(135.3)$ & $(158.46)$ \\
$\left(B r\left[H^{ \pm} \rightarrow \tau v\right], B r\left[H^{ \pm} \rightarrow W^{ \pm} h\right]\right)$ & $(10.0 \% ; 72.0 \%)$ & $(97.3 \% ; 0.34 \%)$ \\
$\left(\operatorname{Br}\left[H^{ \pm} \rightarrow c s\right], B r\left[H^{ \pm} \rightarrow c b\right]\right)$ & $(7.0 \% ; 0.01 \%)$ & $(-;-)$ \\
$\left(B r\left[H^{ \pm} \rightarrow t b\right], B r\left[t \rightarrow H^{ \pm} b\right]\right)$ & $(10.0 ; 0.08 \%)$ & $(0.03 \% ;-)$ \\
\hline \hline
\end{tabular}

Table 2: The best-fit points in Type-I and Type-X 2HDM. The decay width of $\Gamma_{H^{ \pm}}$, cross section $(\sigma[p p \rightarrow$ $\left.t H^{+}\right], \sigma\left[p p \rightarrow H^{-} H^{+}\right]$) and decay branching ratios for charged Higgs are listed, where the decay branching ratios smaller than $10^{-4}$ are neglected. We are fixed $m_{h}=125 \mathrm{GeV}$ and $m_{12}^{2}=m_{h}^{2} \tan \beta /\left(1+\tan ^{2} \beta\right)$

\section{Conclusion}

In this contribution we confront the production and branching fractions of light charged Higgs in 2HDM type-1 and type-X with recent LHC data. Our finding are best-fit as well as some proposal benchmark points with the possibility of investigating then in the next Run-3. Light charged Higgs below the top quark mass still allowed by data even by increasing luminosity up to $300 \mathrm{bf}^{-1}$. Light charged Higgs mass together with $95 \mathrm{GeV}$ is also one of the important chanllenge at the next agenda of LHC communitty.

\section{Acknowledgments}

AA and RB are supported by the grant H2020-MSCA-RISE-2014 no. 645722 (NonMinimalHiggs). This work is also supported by the Moroccan Ministry of Higher Education and Scientific Research MESRSFC and CNRST: Project PPR/2015/6. 


\section{References}

[1] G. Aad et al. [ATLAS Collaboration], Phys. Lett. B 716 (2012) 1 doi:10.1016/j.physletb.2012.08.020 [arXiv:1207.7214 [hep-ex]].

[2] S. Chatrchyan et al. [CMS Collaboration], Phys. Lett. B 716 (2012) 30 doi:10.1016/j.physletb.2012.08.021 [arXiv:1207.7235 [hep-ex]].

[3] J. F. Gunion and H. E. Haber, Phys. Rev. D 67, 075019 (2003) [hep-ph/0207010].

[4] G. C. Branco, L. Lavoura and J. P. Silva, Int. Ser. Monogr. Phys. 103, 1 (1999).

[5] M. Gomez-Bock and R. Noriega-Papaqui, J. Phys. G 32, 761 (2006) [hep-ph/0509353].

[6] A. Arhrib, R. Benbrik, C. H. Chen, J. K. Parry, L. Rahili, S. Semlali and Q. S. Yan, arXiv:1710.05898 [hep-ph].

[7] M. Carena, I. Low, N. R. Shah and C. E. M. Wagner, JHEP 1404 (2014) 015 doi:10.1007/JHEP04(2014)015 [arXiv:1310.2248 [hep-ph]].

[8] J. Bernon, J. F. Gunion, H. E. Haber, Y. Jiang and S. Kraml, Phys. Rev. D 92 (2015) no.7, 075004 doi:10.1103/PhysRevD.92.075004 [arXiv:1507.00933 [hep-ph]].

[9] P. M. Ferreira, R. Santos, M. Sher and J. P. Silva, Phys. Rev. D 85 (2012) 035020 doi:10.1103/PhysRevD.85.035020 [arXiv:1201.0019 [hep-ph]].

[10] J. Bernon, J. F. Gunion, H. E. Haber, Y. Jiang and S. Kraml, Phys. Rev. D 93 (2016) no.3, 035027 doi:10.1103/PhysRevD.93.035027 [arXiv:1511.03682 [hep-ph]].

[11] P. M. Ferreira, J. F. Gunion, H. E. Haber and R. Santos, Phys. Rev. D 89, no. 11, 115003 (2014) [arXiv:1403.4736 [hep-ph]].

[12] P. M. Ferreira, R. Guedes, M. O. P. Sampaio and R. Santos, JHEP 1412, 067 (2014) [arXiv:1409.6723 [hep-ph]].

[13] D. Eriksson, J. Rathsman and O. Stal, Comput. Phys. Commun. 181, 189 (2010) [arXiv:0902.0851 [hep-ph]].

[14] V. D. Barger et al., Phys. Lett. B 324, 236 (1994); J. F. Gunion, et al., Nucl. Phys. B 294, 621 (1987). R. M. Barnett et al., Nucl. Phys. B 306, 697 (1988). J. L. Diaz-Cruz and O. A. Sampayo, Phys. Rev. D 50, 6820 (1994). F. Borzumati et al., Phys. Rev. D 60, 115011 (1999).

[15] D. A. Dicus et al., Phys. Rev. D 40, 787 (1989). A. A. Barrientos et al., Phys. Rev. D 59, 015009 (1999); A. A. Barrientos et al., Phys. Rev. D 63, 015009 (2001); W. Hollik and S. h. Zhu, Phys. Rev. D 65, 075015 (2002); O. Brein et al., Phys. Rev. D 63, 095001 (2001).

[16] Q. H. Cao et al., Phys. Rev. D 69, 075008 (2004).

[17] A. C. Bawa et al., Z. Phys. C 47, 75 (1990). A. A. Barrientos et al., Nucl. Phys. B 568, 305 (2000); A. Krause et al., Nucl. Phys. B 519, 85 (1998).

[18] S. Moretti and K. Odagiri, Phys. Rev. D 55, 5627 (1997). A. Arhrib et al., JHEP 1605, 093 (2016).

[19] S. Dittmaier et al., Phys. Rev. D 77, 115001 (2008).

[20] M. Aoki et al., Phys. Rev. D 84, 055028 (2011)

[21] A. G. Akeroyd et al., Eur. Phys. J. C 77, no. 5, 276 (2017) doi:10.1140/epjc/s10052-017-4829-2 [arXiv:1607.01320 [hep-ph]]. 
[22] M. Aaboud et al. [ATLAS Collaboration], JHEP 1809 (2018) 139 doi:10.1007/JHEP09(2018)139 [arXiv:1807.07915 [hep-ex]].

[23] M. Aaboud et al. [ATLAS Collaboration], Phys. Lett. B 759 (2016) 555 doi:10.1016/j.physletb.2016.06.017 [arXiv:1603.09203 [hep-ex]].

[24] G. Aad et al. [ATLAS Collaboration], Phys. Rev. Lett. 114 (2015) no.23, 231801 doi:10.1103/PhysRevLett.114.231801 [arXiv:1503.04233 [hep-ex]].

[25] G. Aad et al. [ATLAS Collaboration], JHEP 1503 (2015) 088 doi:10.1007/JHEP03(2015)088 [arXiv:1412.6663 [hep-ex]].

[26] G. Aad et al. [ATLAS Collaboration], Eur. Phys. J. C 73 (2013) no.6, 2465 doi:10.1140/epjc/s10052-013-2465-z [arXiv:1302.3694 [hep-ex]].

[27] G. Aad et al. [ATLAS Collaboration], JHEP 1206 (2012) 039 doi:10.1007/JHEP06(2012)039 [arXiv:1204.2760 [hep-ex]].

[28] A. M. Sirunyan et al. [CMS Collaboration], JHEP 1811 (2018) 115 doi:10.1007/JHEP11(2018)115 [arXiv: 1808.06575 [hep-ex]].

[29] V. Khachatryan et al. [CMS Collaboration], JHEP 1512 (2015) 178 doi:10.1007/JHEP12(2015)178 [arXiv:1510.04252 [hep-ex]].

[30] V. Khachatryan et al. [CMS Collaboration], JHEP 1511 (2015) 018 doi:10.1007/JHEP11(2015)018 [arXiv:1508.07774 [hep-ex]].

[31] S. Chatrchyan et al. [CMS Collaboration], JHEP 1207 (2012) 143 doi:10.1007/JHEP07(2012)143 [arXiv:1205.5736 [hep-ex]].

[32] M. Tanabashi et al. [Particle Data Group], Phys. Rev. D 98 (2018) no.3, 030001. doi:10.1103/PhysRevD.98.030001

[33] P. Bechtle, O. Brein, S. Heinemeyer, O. StÃěl, T. Stefaniak, G. Weiglein and K. E. Williams, Eur. Phys. J. C 74 (2014) no.3, 2693 doi:10.1140/epjc/s10052-013-2693-2 [arXiv:1311.0055 [hep-ph]].

[34] P. Bechtle, S. Heinemeyer, O. StÃěl, T. Stefaniak and G. Weiglein, Eur. Phys. J. C 74 (2014) no.2, 2711 doi:10.1140/epjc/s10052-013-2711-4 [arXiv:1305.1933 [hep-ph]].

[35] F. Mahmoudi, Comput. Phys. Commun. 180 (2009) 1579 doi:10.1016/j.cpc.2009.02.017 [arXiv:0808.3144 [hep-ph]]. 